\title{
Propuesta metodológica para la conformación de una carpeta de evidencias de
}

\author{
García Velazco, G. ${ }^{1}$, Méndez Guevara, L. C. ${ }^{2}$ Espinoza Ortega, O. ${ }^{3}$ \\ ${ }^{1}$ Coordinación de la Licenciatura en Informática Administrativa. \\ ${ }^{2,3}$ Docentes adscritos a la Licenciatura en Informática Administrativa y Licenciatura en Ingeniería en Computación \\ Centro Universitario UAEM Valle de Teotihuacán. \\ Cerrada Netzahualcóyotl, s/n. 55955 Axapusco, Estado de México. \\ 'ggarciav@uaemex.mx, ${ }^{2}$ lcmendezg@ uaemex.mx, ${ }^{3}$ oespinozao@uaemex.mx
}

Fecha de recepción: 17 septiembre 2014

Fecha de aceptación: 11 de diciembre 2014

Resumen. Este documento establece una propuesta metodológica para la conformación de una carpeta de evidencias de desempeño, cuyo propósito es la evaluación del estudiante, ya que representa un instrumento que se va conformando día a día, con las actividades que se realizan en clase o extra clase de temas que el docente planea dentro del marco de una unidad de aprendizaje y que el estudiante ejecuta.

La carpeta de evidencias permite dar seguimiento al proceso de evaluación sumativa del estudiante, permitiendo centrarse en el desarrollo de las competencias del estudiante haciéndolo más autodidacta, al aprender de forma autónoma y analizar su propio aprendizaje. Al docente le permite ver la forma en la que estableció sus actividades y comparar si los resultados que obtuvo fueron los esperados, lo que le permitirá revalorizarlos y hacer ajustes a sus programas con el objetivo de crecer y mejorar en esa área del conocimiento.

Palabras Clave: Docencia, Competencias Profesionales, Evidencias de Desempeño, Carpeta de Evidencias.

Summary. This document sets out a methodology for the creation of a folder evidence of performance, aimed at assessing the student, as it represents an instrument that is taking shape day by day, with activities carried out in class or extra-class topics that teachers plan within the framework of a learning unit and the student run. The folder of evidence allows to monitor the process of summative student assessment, allowing focus on developing student skills making it self, to learn independently and analyze their own learning. Teaching lets you see how it established its activities and compare whether the results obtained were as expected, allowing you to revalue and make adjustments to their programs in order to grow and improve in that area of knowledge.

Keywords: Teaching, Professional Skills, Performance Evidence, Evidence Folder.

\section{Introducción}

La carpeta de evidencias es una herramienta de apoyo, que sirve al docente como medio de evaluación para establecer las competencias logradas por los estudiantes en una unidad de aprendizaje específica, y determinar el grado de aprendizaje que cada uno tuvo en particular. Al respecto, Cano (2003) menciona, "una carpeta es un contenedor de diversos documentos, donde se encuentran sus esfuerzos, progresos y consecuciones, es el registro del aprendizaje de un profesional".

La carpeta de evidencias de forma impresa o electrónica, permite centrar el aprendizaje en el estudiante y demostrar la forma en la que él desarrolló su conocimiento y competencias en el transcurso del periodo escolar.

\subsection{Justificación}

En la educación tradicional, generalmente el docente evalúa sin la participación del alumno, adoptando un rol pasivo como emisor de información, es decir los aspectos de enseñanza y evaluación le confiere únicamente a él, de tal manera que considera como más relevante el resultado final, dando menor importancia al proceso enseñanza-aprendizaje. 
Para dar un giro en el proceso de enseñanza aprendizaje, se han modificado algunas de las funciones que anteriormente eran propias de un profesor. Actualmente se busca que la labor del docente sea guiar, orientar, facilitar y mediar los aprendizajes significativos en los alumnos.

El objetivo de las nuevas estrategias de aprendizaje y evaluación es facilitar el proceso de "Aprender a aprender", que pretende lograr que el alumno aprenda de forma autónoma y activa, independientemente de las situaciones de enseñanza. De esta manera, el docente debe adoptar estrategias diversas, según las necesidades e intenciones deseadas que le permita atender a los diferentes estilos y ritmos de aprendizaje de los alumnos.

Una evaluación guiada en la enseñanza por competencias, debe incluir diversas técnicas e instrumentos que permitan realizarla en diferentes dimensiones: conceptuales, procedimentales y actitudinales. Por lo cual se propone la "carpeta de evidencias de desempeño" que es una colección de documentos con ciertas características que tienen como propósito evaluar el nivel de aprendizaje que se ha adquirido.

Lo anterior pretende lograr una mejora en la adquisición de conocimiento y la evaluación del alumno mediante una carpeta de evidencias, en donde no se excluyen las pruebas objetivas (exámenes tradicionales) sino que se enriquece con productos elaborados por los alumnos y que reflejan los esfuerzos, avances y logros a lo largo del curso.

La carpeta de evidencias permite al alumno participar en la evaluación de su propio desempeño, recopilando información en donde se manifiesten los avances de los aprendizajes conceptuales, actitudinales y procedimentales de los alumnos, mediante el portafolio, el profesor puede llevar un registro objetivo y documentado de los avances de los alumnos, logrando en ambos un roll activo que promete un avance significativo en el proceso de enseñanza-aprendizaje.

\subsection{Estado del arte}

El estado del arte en el desarrollo de carpetas de evidencia de desempeño, lo podemos centrar en dos puntos, la formación y desarrollo profesional docente y como una herramienta de apoyo para evaluar el aprendizaje, las cuáles se plantean a continuación.

\section{Formación y desarrollo profesional docente}

El uso de la carpeta de evidencias forma parte de la reflexión del propio trabajo docente, la cual tiene una finalidad de evidencia formativa y de mejora continua del proceso de enseñanza, al permitir observar los aciertos y fallas de su propia planeación. Al respecto:

- Cano e Ibernon (2003), crean una carpeta de evidencias electrónica, que incluía contenidos y guías, adicionando dos columnas, la primera con interrogantes acerca de reflexiones del quehacer docente y la segunda con evidencias para responder las interrogantes reflexionadas, buscando con ello crear una cultura docente colaborativa reflexiva en el procesos de enseñanza - aprendizaje.

- Predes y Sanchéz (2008) mencionan que la carpeta de evidencias no solo ofrece una reflexión crítica de la actividad docente, también es una herramienta de evaluación entre pares, generando una mejor comunicación a través de las experiencias recabadas, al ser una evidencia documentada de la enseñanza, en función de los objetivos y contextos específicos.

- Para Sánchez (2011) la carpeta de evidencia es parte de la exigencia de calidad que lleva aparejada la renovación docente y propone la utilización de un índice, una introducción, la inclusión de los temas centrales seleccionados por el alumno y el cierre con la síntesis del aprendizaje

\section{Herramienta para evaluar el aprendizaje}

El uso de la carpeta de evidencias como parte de las herramientas de evaluación de los saberes y competencias de los alumnos. Al respecto:

- Sánchez (2011) menciona que la carpeta de evidencia permite conocer, evaluar, autoevaluar y retroalimentar todos los materiales que intervienen en el proceso formativo como son: artículos, textos legales, documentos multimedia, mapas, planos, fotografías, entre otros y los criterios a utilizar dependen de la unidad de aprendizaje 
en particular, generando transparencia de los criterios de evaluación. Lo que aporta evidencia acerca de la capacidad de decidir, comunicar y reflexionar sobre su propio aprendizaje.

- Cano (2003) propone que la integración de la carpeta debe ser enriquecida con otras fuentes e incluir tres elementos: reflexiones sobre los aprendizajes en clase, materiales de otros que refuercen el conocimiento y la demostración del producto de aprendizaje mediante ensayo o trabajos creativos. Para la integración general incluye la estructura que se muestra en la figura 1.
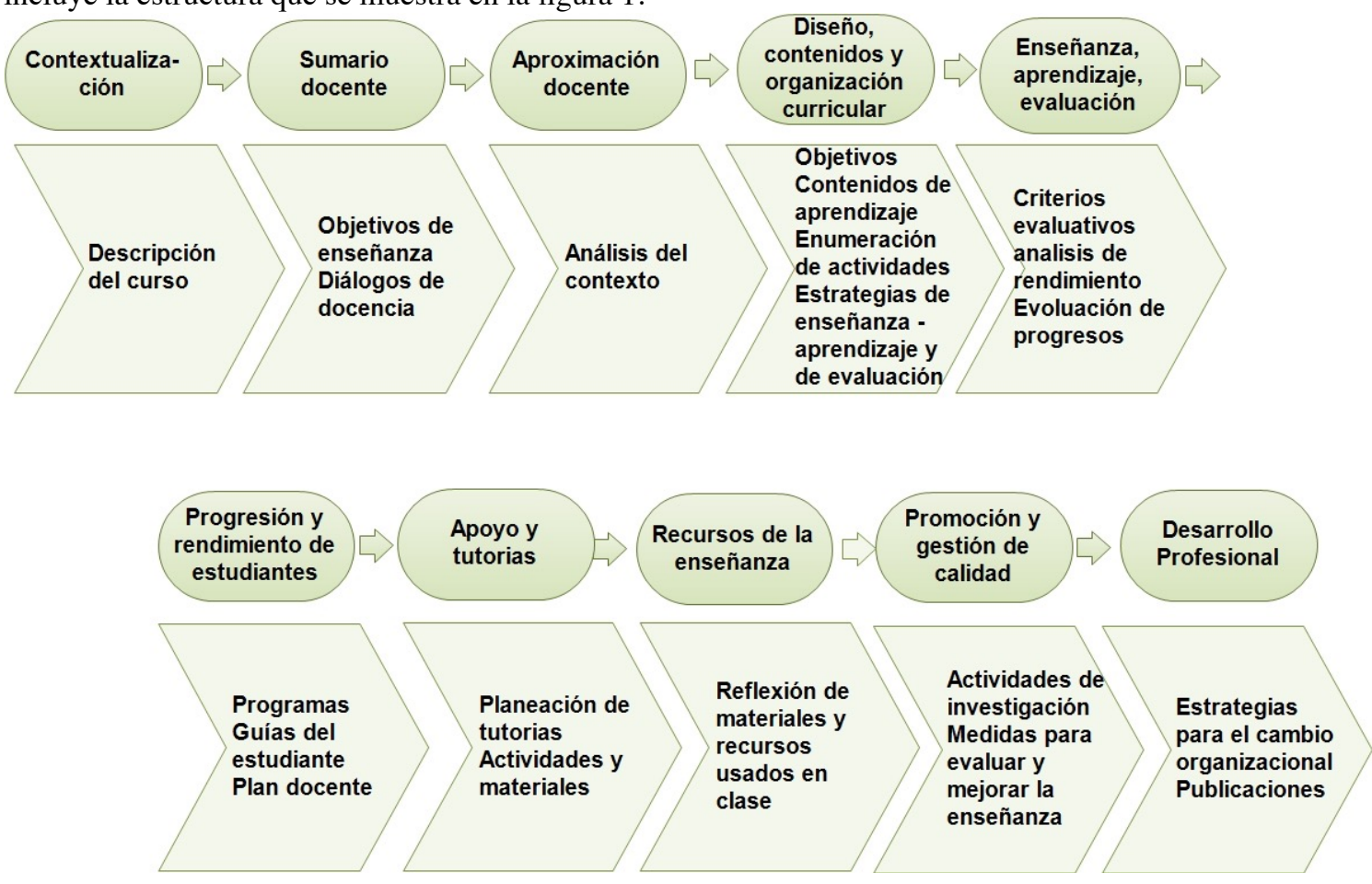

Fig. 1 Estructura de la carpeta de evidencias (Elaboración propia, fuente: Cano (2003))

\subsection{Educación basada en competencias}

La educación basada en competencias (EBC) es una nueva orientación educativa que pretende dar respuesta a la sociedad de la información, donde todos los rubros, tienen la misma importancia, para alcanzar las metas educativas además de estar vinculados entre sí para el logro de las competencias (Vázquez, 2001).

La EBC se centra en las necesidades del estudiante, buscando fortalecer su aprendizaje, destrezas y valores, traduciendo el proceso del "conocer" en un "saber", es decir, transformando la teoría en respuestas a problemas del mundo real en diversos escenarios.

Vázquez (2001) menciona, que la EBC se determina por una manifestación de evidencias en el nivel de aprendizaje del conocimiento, desarrollo de las habilidades y valores. Además de actitudes y valores que se manifiestan en un contexto de ejecución de una tarea (Gutiérrez, 2005).

En la EBC la conformación de las evidencias toma suma importancia, a través de ellas podemos observar la construcción del proceso de aprendizaje y desarrollo de competencias del estudiante, integrado en una carpeta de evidencia de desempeño, que sirve como una herramienta de evaluación para el docente.

\subsection{Carpeta de evidencias}

Según Cano (2003), la carpeta de evidencias recoge los esfuerzos y resultados del docente para mejorar su enseñanza y su profesionalización además de ser una herramienta que le sirve para ser evaluado o para promocionar laboralmente, en Cano y Imbernon (2003) se agrega que está exhibe los logros del creador y sus competencias profesionales. 


\subsection{Utilidad de la Carpeta de evidencias}

La carpeta de evidencias de desempeño es una herramienta que se ha adaptado al proceso de la enseñanza (ver, figura 2) que sirve para medir los conocimientos, la forma en la que los adquirió y el desempeño de las competencias profesionales del estudiante, pero también sirve al docente para observar el transcurso de la enseñanza, recoger las metas alcanzadas, compararlas contra las esperadas y poder implementar mejoras en el proceso educativo, además de servirle como una herramienta de evaluación de competencias de desempeño docente. Por lo anterior, podemos decir que este instrumento sirve al docente de evidencia tanto al estudiante, como al docente.

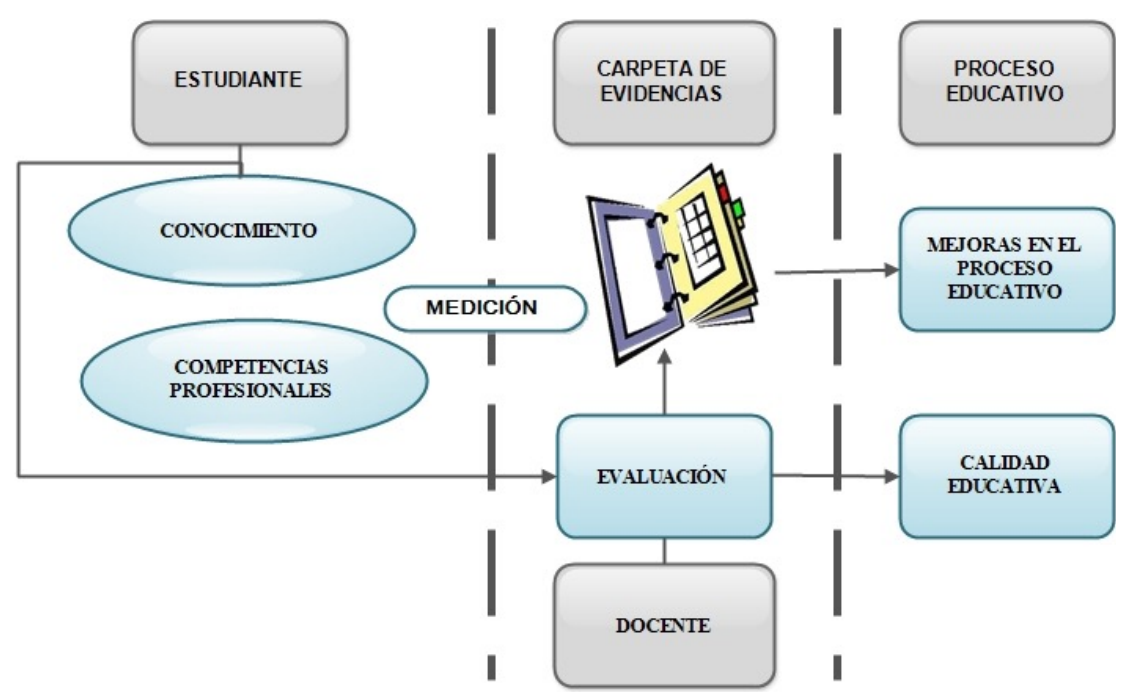

Fig. 2. Utilidad de la carpeta de evidencias en el proceso educativo (Fuente: Propia).

\subsection{Organización y diseño de la carpeta de evidencias}

Cuando se diseña, se organiza y se crea un programa de formación mediante una carpeta de evidencias, se realiza un acto teórico, puesto que será la teoría que se sostenga sobre la enseñanza y el aprendizaje (Shulman, citado en Cano y Imbernon, 2003).

El docente de cada unidad de aprendizaje (UA) indicará a sus estudiantes los conceptos, ejemplos, ejercicios y prácticas relacionados a los criterios de desempeño de cada UA, que conformarán la carpeta de evidencias y determinará la forma en la que estos deberán ser presentados (mapas conceptuales/mentales, ensayos, reportes, entre otros), de tal forma que se demuestre la comprensión y el aprendizaje significativo del estudiante.

La carpeta de evidencias (ver, figura 3) contendrá, los contenidos y los instrumentos de evaluación (rúbricas, listas de cotejo y/o guías de observación) con los criterios que permitan demostrar y medir las competencias alcanzadas por los estudiantes a lo largo y al final de cada unidad de aprendizaje. Así, cada estudiante conformará su carpeta de forma impresa o electrónica durante el desarrollo de la unidad de aprendizaje, con revisiones continúas por parte del docente y con cortes a cada evaluación, permitiéndole demostrar su aprendizaje y las competencias adquiridas.

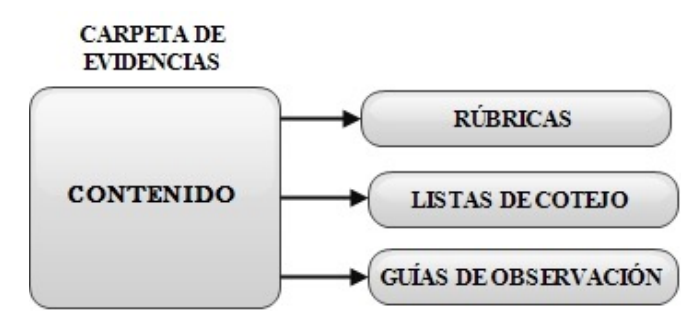

Fig. 3. Integración de la carpeta de evidencias (Fuente: propia). 


\section{Metodología propuesta para la integración de la carpeta de evidencias.}

\subsection{Metodología de la carpeta de evidencias}

Buscando estandarizar las carpetas de evidencias así como lograr la identificación institucional del estudiante y docente, se establecen una propuesta para la integración de la misma, la cual se muestra en la Figura 4.

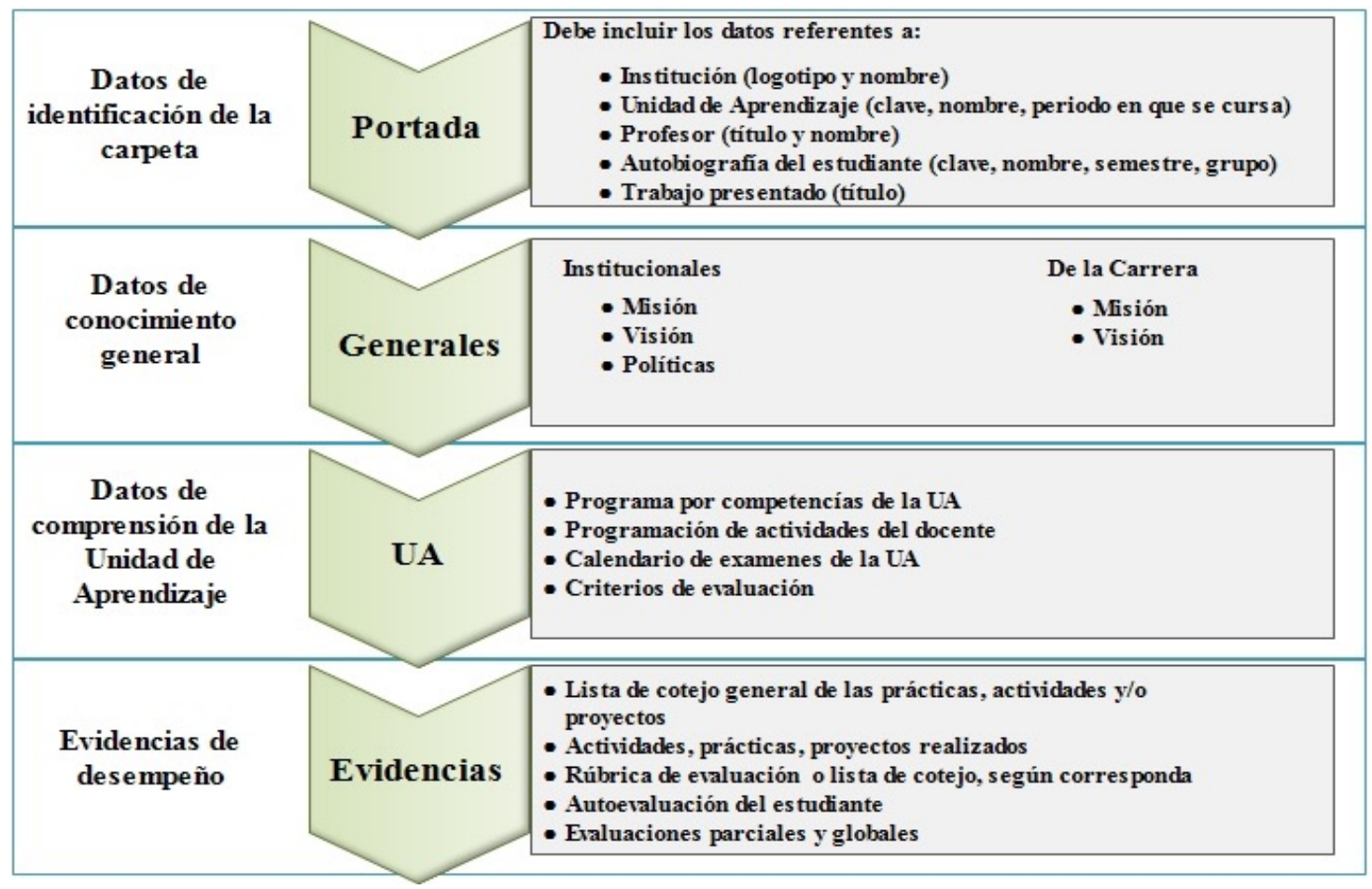

Fig. 4. Metodología para el establecimiento de la carpeta de evidencias (Fuente: Propia).

La metodología propuesta para el establecimiento de la carpeta de evidencias, se describe a continuación:

1. La portada de presentación de la carpeta debe incluir los datos que identifiquen plenamente el trabajo presentado por el estudiante, como son la institución a la que pertenece, unidad de aprendizaje que cursa, docente que imparte, la autobiografía del estudiante y el título del trabajo.

2. Los datos generales, tanto institucionales, del programa educativo y carrera al que está adscrito el estudiante, darán un panorama general de lo que se pretende lograr en su formación integral, ayudando a fomentar una identidad institucional.

3. Los datos de comprensión de la unidad de aprendizaje, para qué el docente y el estudiante no los pierdan de vista, esta debe incluir, el programa por competencias de la unidad de aprendizaje, y el programa de actividades del docente para corroborar en cualquier momento del proceso que se cumple con los objetivos establecidos y poder darle seguimiento, debe incluir los criterios de valor que esté asigna a cada actividad para su conocimiento y aplicación.

4. Las evidencias de desempeño, consisten en una lista de cotejo de las actividades especificadas por el docente (ver Figura 5), quien marcará si fueron incluidas o no, detrás de esta, se encontrará cada actividad, práctica o proyecto documentada con su respectiva rúbrica o lista de cotejo evaluada, al final de ellas se incluirá la propia autoevaluación del estudiante, la cual puede ser un cuestionario estructurado o no estructurado, así como las evaluaciones ordinaria. 


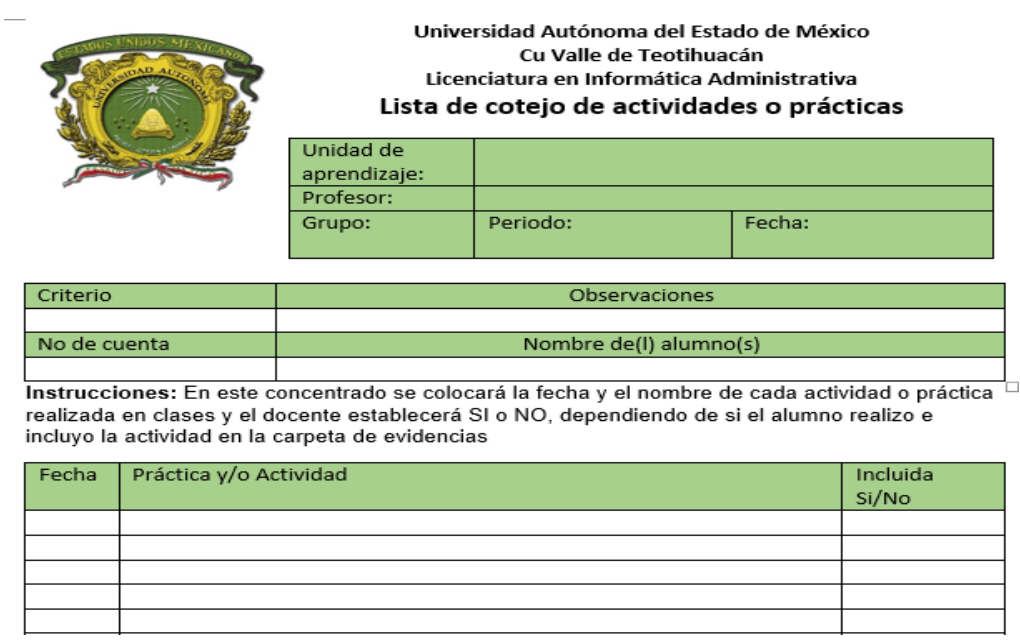

Fig. 4. Ejemplo de una lista de cotejo de actividades o prácticas (Fuente: propia).

\subsection{Implementación de la carpeta de evidencias}

La implementación de la carpeta de evidencias en cada una de las unidades de aprendizaje, se puede realizar siguiendo el proceso que se plantea a continuación:

1) Planeación.

a. El docente establece la programación semestral de su unidad de aprendizaje, la cual incluye los temas de las unidades, fechas para las actividades, ejercicios y/o prácticas planeadas y exámenes, así como el valor que tendrá cada una y el instrumento que utilizará para evaluarlas (rúbrica, lista de cotejo y/o guía de observación), previo al inicio de clases.

b. El docente entrega a la coordinación de carrera la programación semestral.

c. El primer día de clases el docente presenta la unidad de aprendizaje, la programación de actividades, bibliografía y entrega las rúbricas, lista de cotejo y/o guía de observación que usará durante el semestre a los alumnos, para que ellos estén en conocimiento de los objetivos, temas, la forma de trabajo, evaluación y periodos de entrega.

2) Ejecución y control.

a. El alumno estructura la carpeta de evidencias de cada una de sus unidades de aprendizaje siguiendo la metodología y el orden propuesto para ello.

b. Durante el desarrollo del semestre el alumno hace entrega al docente de cada una de sus actividades, ejercicios y prácticas, en las fechas establecidas para ello e incluye las rúbricas correspondientes.

c. El docente evalúa y coloca las observaciones correspondientes en la rúbrica, lo que sirve de retroalimentación al alumno, quien puede complementar la información en base a lo observado.

d. El alumno coloca la actividad y rúbrica en la carpeta de evidencias

e. Al final de cada parcial el docente revisa las carpetas de evidencia y observa el avance de cada alumno, lo que le da un parámetro para evaluar el aprendizaje y competencias desarrolladas en el alumno.

f. El docente puede detectar en cualquier momento, las áreas del conocimiento que deben ser reforzadas, en base al desempeño mostrado por los alumnos en las carpetas y ejercer los mecanismos de control que requiera para reforzar el aprendizaje.

3) Cierre.

a. Al final del semestre el alumno entrega en forma electrónica la carpeta al docente y él se queda con la carpeta física, el docente entrega una copia de ésta a la coordinación de carrera, lo que permite:

i. Al docente, observar en la carpeta de evidencias de sus alumnos, el desarrollo de su propio trabajo e implementar estrategias para mejorar su función docente.

ii. Al alumno ver el desarrollo de sus competencias y evaluar la forma en que construyo su propio conocimiento. 
iii. A la coordinación de carrera le permite:

1. Ver el desempeño y dominio que tiene el docente acerca de la unidad de aprendizaje y aunado a otros mecanismos como la evaluación docente y la apreciación estudiantil, analizar las deficiencias didácticas y pedagógicas que se presenten para tomar las medidas necesarias y proponer cursos en función de que estas sean cubiertas.

2. Conocer el rigor que emplea el docente en la evaluación de sus actividades programadas.

3. Tener un mecanismo de evidencia para la evaluación de los organismos acreditadores

\subsection{Roles}

En la conformación de la carpeta de evidencias se tienen dos roles principales:

- Docente. Como guía durante el periodo de construcción del conocimiento y competencias, pero también funge como evaluador del proceso, a través de la revisión de las diferentes actividades programadas, con el apoyo de los criterios plasmados en las rúbricas o listas de cotejo correspondientes y la aplicación de las evaluaciones ordinarias.

- Estudiante. Creador de la carpeta de evidencias de cada UA, a través de la metodología establecida y también funge como evaluador de su propio conocimiento, y adicionalmente realiza su propia autoevaluación.

\subsection{Escenario}

A continuación, se describe en la Tabla 1 un escenario donde el profesor usa un método tradicional para evaluar a un estudiante, y en la Tabla 2 se observa un modelo EBC que usa como herramienta de apoyo la metodología propuesta de carpetas de evidencia.

Tabla 1. Escenario de evaluación tradicional (fuente: propia).

\section{Docente}

- Programa actividades y fechas de entrega y las hace de su conocimiento al estudiante en cualquier parte del programa.

- $\quad$ Explica verbalmente en que consiste la actividad, lo que puede generar dudas en estudiante al respecto.

- Evalúa las actividades de los estudiantes en libreta, hojas sueltas, archivos electrónicos.

- La recopilación de firmas y calificaciones de trabajo es más laborioso por la diversidad de medios en las que se encuentra.

- Evalúa las actividades basado en criterios propios, formulados en el momento de revisión y estos son la base de la calificación que otorga.

- $\quad$ Los criterios otorgados por el profesor en las actividades no son unificados, varían en base al tamaño del trabajo, faltas de ortografía, desempeño en clase del estudiante.

\section{Estudiante}

- Realiza y entrega las actividades conforme el docente las indica.

- Desconoce los criterios que el docente ocupará para evaluar cada actividad.

- Falta de conocimiento sobre lo que el profesor espera de la actividad.

- Insatisfecho con la calificación obtenida, debido a que:

o Desconoce el criterio empleado por el profesor.

- Sólo puede valorar su trabajo al compararlo con el de sus compañeros.

Tabla 2. Escenario de evaluación basado en un modelo de competencias, usando carpetas de evidencias (fuente: propia). 
- Programa actividades y fechas de entrega y las hace de su conocimiento al estudiante al inicio del programa.

- Explica en que consiste la actividad, además de tener la planeación por escrito y la da a conocer.

- Establece criterios y valores generales de evaluación para cada actividad.

- Es imparcial, ya que usa una herramienta base como apoyo a la evaluación.

- Recopila la carpeta de evidencias con el concentrado del proceso de enseñanza - aprendizaje.
- Conoce las actividades y fechas de entrega de cada actividad.

- Observa en las rúbricas los criterios que el docente ocupará para evaluar cada actividad.

- $\quad$ Sigue los criterios con mayor exactitud referentes a lo que el docente espera recibir de una actividad.

- $\quad$ Satisfecho con la calificación obtenida, al saber los criterios que cumplió y recibir la retroalimentación de los que faltaron o estuvieron inconclusos.

\section{Conclusiones y trabajos futuros}

Al aplicar la metodología propuesta se obtendrán resultados favorables en la formación de los estudiantes, ya que permite de manera equitativa y justa, asignar una evaluación del desempeño de estos.

La aplicación de la carpeta de evidencia servirá para contar con información organizada y estructurada que permita observar los resultados del proceso de aprendizaje de forma más fácil, detectando las áreas del conocimiento que deben ser reforzadas.

El docente puede evaluar el resultado final de la unidad de aprendizaje, así como el proceso de cada estudiante de forma particular, y puede evaluarse a sí mismo en el desarrollo de sus propias competencias docentes.

El estudiante mejora sus técnicas de aprendizaje al observar el desarrollo de su propio proceso, evalúa su propio aprendizaje, alcanza promedios más altos y se encuentra satisfecho con el nivel de competencias desarrolladas.

El integrar la carpeta de evidencias en todas las unidades de aprendizaje, traerá como beneficio el contar con una herramienta que sirva al profesor para una evaluación de sus competencias docentes, así como para los procesos de acreditaciones y certificaciones de los diversos organismos, al tener documentación organizada de forma pertinente.

Como trabajo futuro queda la propuesta de adaptación de la carpeta de evidencias a las diferentes unidades de aprendizaje de cada docente en particular, así como la reflexión y autoevaluación del propio desarrollo docente en las UA, así como la búsqueda del mejoramiento en el desarrollo de sus propias competencias, así como la elaboración de una plataforma para la conformación de carpetas de evidencia digitales.

\section{Referencias}

1. Cano, E. (2003). La carpeta docente como instrumento formativo. Gairín, J. y Armengol, C., Estrategias de formación para el cambio organizacional. Barcelona: CISS-PRAXIS.

2. Cano, E., \& Imbernon, F. (2003). La carpeta docente como instrumento de desarrollo profesional del profesorado universitario. Revista Interuniversitaria de formación del profesorado, 17(2), 43-51.

3. Cano, E. (2003). La carpeta docente como instrumento formativo. Gairín, J. y Armengol, C., Estrategias de formación para el cambio organizacional. Barcelona: CISS-PRAXIS.

4. Gutiérrez, O. (2005). Educación y entrenamiento basados en el concepto de competencia: Implicaciones para la acreditación de los programas de Psicología. Revista Mexicana de Psicología, 22, número monográfico especial, pp. 253-270.

5. Mellado Hernández, María Elena. "Portafolio en línea en la formación inicial docente." Revista electrónica de investigación educativa 12.1 (2010): 1-32.

6. Pastor, V.M.L. (2009) Evaluación formativa y compartida en Educación Superior: Propuestas, técnicas, instrumentos y experiencias, Narcea, S.A. de Ediciones, Madrid, España 
7. Predes, M. y Sánchez M (2008), El portafolio electrónico: posibilidades para los docentes, Pixel - Bit Revista de Medios y Educación, Universidad de Murcia, España, No 32 Marzo 2008 pp. 21- 34

8. Sánchez, M. R. (2011). Metodologías docentes en el EEES: de la clase magistral al portafolio. CONSEJO DE REDACCIÓN, 83.

9. Vázquez, Y. A. (2001). Educación basada en competencias. Educar: revista de educación/nueva época, $16,1-29$. 